\title{
Brain Tumour Classification by Machine Learning Applications with Selected Biological Features: Towards A Newer Diagnostic Regime
}

\author{
Krishnendu Ghosh ${ }^{1, \#}$, Jayanta Kumar Chandra, ${ }^{2, \#}$ and Anirban Ghosh ${ }^{1,3, *}$ \\ ${ }^{1}$ Immunobiology Laboratory, Department of Zoology, Panihati Mahavidyalaya (West Bengal State University), \\ Barasat Road, Sodepur, West Bengal, India \\ ${ }^{2}$ Department of Electrical Engineering, Ramkrishna Mahato Government Engineering College, Purulia, West \\ Bengal, India \\ ${ }^{3}$ Department of Zoology, School of Sciences, Netaji Subhas Open University, West Bengal, India
}

\begin{abstract}
Histopathologically classified low-grade brain tumours show overlapping biological characteristics making them difficult to distinguish. In the present study low-grade brain tumour patient samples of three different histopathological types have been trained through machine learning technique using selected features for its classification. We used specifically the fundamental proliferation, invasion, macrophage infiltration triangle of cancer hallmark with propidium iodide $(\mathrm{PI})$ marked cell-cycle, Ki67 marked proliferative indexing, invasion with MMP2 expression and presence of macrophage/microglia by silver-gold staining, CD11b+and Iba1+ cell presence as biological parameters. These parameters when trained with proper machine learning protocol through extraction of underling features and represented in a 2D perceivable space are found capable of distinguishing the tumour types. Extracted features from such parameters in a six-dimensional featured space were trained through statistical learning theory while support vector machine (SVM) maximizes their predictive precision. The leave one out (LOO) cross validation process was applied to judge the accuracy of training followed by auto-encoder (AE) to reduce feature dimension at two which is visually perceptible. From the biological features quantified with standard methods it was found impossible to demarcate the three types of low grade brain tumours. However, after training through SVM and LOO cross validation when the sixdimensional featured space had been reduced into two-dimension using $A E$, the combined output of the features showed clear zonation in that $2 \mathrm{D}$ space. This indicates that the overlapping biological characteristics of these tumour types, when trained through proper support vector machine and reduced from multiple to two dimensional space provides a clear patho-clinical classification edge using a combination of common biological features. Hence, machine learning applications may potentially be used as a complementary diagnostic protocol with the conventional practice.
\end{abstract}

Keywords: Brain tumour, Astrocytoma, Meningioma, Ependymoma, Support Vector Machine (SVM), Auto-Encoder $(\mathrm{AE})$.

\section{INTRODUCTION}

Cancer, the second most global cause of mortality, is the abnormal cellular proliferation with loss of contact inhibition, escaping programmed cell death, avoiding cellular senescence, invasive metastasis, neoangiogenesis, self-sufficient metabolism and acquiring immune-tolerance $[1,2]$. From the second half of the last century it has become one of the most highly rated and highly funded areas of research for its massive impact on the health and life-expectancy of human population [3]. Survey by IACR documented 7\% of cancer cases worldwide are from India. Among all cancer types CNS related tumours always receives some special attention as it is associated to the central controlling unit of our body. Primary brain and spinal cord tumours, one of the dreaded type of neoplastic notions, affect nearly 700,000 persons in US

*Address correspondence to this author at the Department of Zoology, School of Sciences, Netaji Subhas Open University, DD-26, Salt Lake, Sector - I, Kolkata - 700064, West Bengal, India; E-mail: aghosh06@gmail.com, anirbanghosh@wbnsou.ac.in

${ }^{\#}$ K.G and J.C both contributed equally as first author encompasing $36.6 \%$ of meningioma and $24.7 \%$ glioma as of January 2018 according to American Brain Tumour Association. Ependymoma on the other hand represents $5 \%$ of all glioma among which spinal myxopapillary ependymoma of WHO grade I represents $90 \%$ of all spinal tumours [4-7].

One of the major problems in medical cancer research, particularly in this region, is the lack of specific information or proper data repertoire mostly due to lesser accessibility of cancer samples for biomedical analysis and thereafter proper data mining and preserving as reference data sets at tissue protein level expression. But for early diagnosis and prognostic measures important feature extraction and validation of such features as distinctive markers of a specific type of tumours is a very important step, which can only be accomplished by the selection of relevant feature sets and a properly trained classifier working on those feature sets to identify the given tumour classes.

Presently, the use of machine learning in the field of biomedical research have reached an extraordinary 
level and spreads in multiple dimensions. This helps in interpretation of large and complex data sets derived from genomic, proteomic, metabolomic and microbiome analysis and also become a handful tool for systems biology approach $[8,9]$. Here, learning of a machine through the machine learning technique means the training of the system for tumor classification using appropriate features from tumour samples of known tumour classes. In the proposed technique six important biological features from real samples of three different low-grade brain tumours, named intracranial diffuse astrocytoma of WHO grade II (eventually considered to be low grade astrocytic tumour as grade I astrocytoma is seemingly rare and literally asymptomatic), intradural fibrous meningioma and extradural spinal myxopapillary ependymoma belonging to $\mathrm{WHO}$ grade I, were particularly selected to basically encircle the fundamental proliferationinvasion-inflammation triangle of cancer [1, 2] are used as the candidate features.

Thus, in this case the tumour data samples may be viewed as the data points in a six dimensional feature space which are required to be separated to fall into the specific class of the tumours by placing hyperplanes and parameterized by weight vectors. The support vector machine (SVM) can accomplish this task [10] by maximizing the predictive accuracy, using the statistical learning theory [11]. The leave one out (LOO) cross validation process has been applied to judge the training and test case accuracy, under the constraint of limited number of data samples. As it is difficult to visualize and realize a space more than 3 dimensions, the auto encoder (AE) has been used to reduce multidimensional space to 2 dimensions without any loss of information, related to classification [12].

\section{MATERIALS AND METHODS}

The process of biochemical analysis was carried out on post-operative human CNS tumour samples obtained from Department of Neurosurgery, Bangur Institute of Neurosciences (BIN), Institute of Post Graduate Medical Education and Research (IPGME\&R), India, with the approval taken from the ethical committee. For statistical analysis MATLAB 2018a was used.

\subsection{Biological Analysis}

\subsubsection{Histopathological Grading}

Brain tumour tissue samples from patients were readily processed with alcoholic dehydration, paraffin embedding, microtome sectioning and standard eosine-hematoxylin staining, observing and documenting under microscope as described in Ghosh et al., 2016 for histopathological gradation and identification of the tumour samples.

\subsubsection{Immunohistochemical Analysis for Immune- Cells and Proliferating Cells}

For the specific silver-gold (SG) staining brain tumour tissue samples were stained in ammoniacal Silver Carbonate solution and stained subsequently in Gold Chloride solutions followed by fixation in sodium thiosulphate, observed and recorded under microscope as detailed in literature $[13,14]$. After primary preparation, tissue slides were incubated overnight with primary non-conjugated Ki67 antibody (Santacruz, USA) to detect proliferating cell nucleus. Then treated with HRP-conjugated secondary antibody (Santacruz, USA) and DAB (SRL, India) in buffered $\mathrm{H}_{2} \mathrm{O}_{2}$ with $0.5 \%$ cupric sulfate solution in dark, followed by counterstaining with hematoxylin, air dried and observed. Photographs were analysed with software (NIS Element-BR, Nikon, Japan) to record the presence of electron dense macrophage/microglia in samples as number of SG stained cells per $\sim 50,000 \mu \mathrm{m}^{2}$ area per samples. Similarly, Ki67 index which reflects proportional presence of Ki67 positive nucleus (i.e., number of Ki67 positive cells/Ki67 negative cells) in each sample field of the brain tumour samples were calculated and tabulated for further analysis.

\subsubsection{Immunoflurescence Analysis of Immune-Cell Infiltration}

Tissue samples preserved in paraformaldehyde and tissue slides were prepared, heat fixed and stained with specific monoclonal antibodies for staining with specific receptors of our targets on specific cell types or for intracellular proteins. Primary conjugated CD11b-FITC antibody (BioLegend, USA) was used for brain monocyte/macrophage cells, primary non-conjugated Iba1 mAb with PE-conjugate (Abcam, USA) was used as brain macrophage/microglia marker. Immunofluorescence staining of the tissues with CD11b and Iba1 markers were observed, photographed and analysed with Nikon TS100-F Eclipse Microscope with Epi-fluorescence attachment using Epi-FL filter Block B-2A for Alexa Fluor ${ }^{\circledR}$ 488/FITC and Epi-FL filter Block G-2A for PE/TRITC (Nikon Corp., Japan). Photographs were captured with CCD camera, processed, analysed and documented with Software as mentioned in earlier section. 


\subsubsection{Flowcytometric Analysis of Cell Cycle from Parafinized Tissue Blocks}

To analyse the cell cycle phase distribution which is an important parameter to identify proliferating status of tumour samples the tissues were prepared from paraffinized tissue blocks after treating with xylene, centrifugation and passing through graded alcohol and PBS [15]. Incubation in citrate buffer and digestion in trypsin-EDTA solution (Sigma Aldrich, USA) with PBS wash and passage through 70 um mesh followed by RNase treatment and Propidium lodide (PI) incubation stained nuclear DNA. These PI stained cells were taken for reading the fluorescence in BD Accuri C5 (BD Biosciences, USA) and analysed for DNA content at different stages of cell cycle and proliferative cell population were measured as found in $S \& G_{2} M$ phases.

\subsubsection{Flowcytometric Analysis of Invasive Factor}

Fresh brain tumour samples were collected, prepared and plated for culture with $1.5 \times 10^{6} \mathrm{cells} / \mathrm{ml}$ in $10 \%$ FBS-1X DMEM with antibiotic-antimycotic solution in $37^{\circ} \mathrm{C}-5 \% \mathrm{CO}_{2}$ humidified incubator (New Burnswick, Eppendorf, UK). Cultured cells were taken and treated with permeabilization blocking buffer and after washing incubated with monoclonal antibody for MMP2 (Novus Biologicals, USA) with PE-conjugated secondary antibody (Abcam, USA) for detecting total MMP2 protein expression. The expression was analysed from fluorescence reading taken in BD-FACS Verse flowcytometer (BD Biosciences, USA), analysed by FACS Verse Suit 1.0 (BD Biosciences, USA) with graphical representation and quantification of median fluorescence.

\subsection{Mathematical Analysis}

\subsubsection{Classification of Glioma Samples by Multi Class Support Vector Machines (Multi-Class SVM)}

SVM utilizes supervised learning technique to learn a separating hyper-plane from the training data samples with high confidence, which is represented by the weight vector [16]. As confidence becomes high, placement of support vectors i.e., the closest training data points of either class from the separating hyperplane are placed optimally, making the performance of the classifier better. Multi class SVM extends this concept for the multi-class classification.

Multi class SVM classifier attempts to minimize the following loss function [17],

$$
L=\left\{\sum_{k} \sum_{l} \lambda\left\|W_{k l}\right\|^{2}+\frac{1}{N} \sum_{i} \sum_{j \neq t} \max \left[0,\left(f\left(X_{i}, W\right)_{j}-f\left(X_{i}, W\right)_{t}+\Delta\right)\right]\right\}
$$

Where, $X_{i}$ denotes $i^{\text {th }}$ element of input feature vector of $j^{\text {th }}$ training sample $X^{j} \in \Re^{(1 \times p)}$ suffering from Glioma class $t$. There are $\mathrm{N}$ such training samples. $W \in \mathfrak{R}^{(N \times p)}$ is the weight matrix, $\lambda$ is a scalar multiplier and $\Delta$ is another scalar value indicating the confidence of classification. $f($.) denotes a nonlinear function, mapping $\left(W^{T} \cdot X^{j}\right)$ to a manageable range. The above equation not only ensures proper classification of the training data but also with a high pre specified confidence value, which assures even better performance of the system for the unseen test data. $\lambda\left\|W_{k l}\right\|^{2}$ and $\frac{1}{N} \sum_{i} \sum_{j \neq t} \max \left[0,\left(f\left(X_{i}, W\right)_{j}-f\left(X_{i}, W\right)_{t}+\Delta\right)\right]$ are known as the regularization and data loss terms respectively. To understand the performance of the system for test data LOO cross validation technique is applied in which out of $N$ numbers of training data $(N-1)$ are used for training and remaining one is used for testing purpose in a single epoch. The process is repeated for $N$ times to ensure that all the data in the training data set has been tested blindly for once. Finally the average error is calculated to understand the performance of the system [18].

\subsubsection{Reduction of Feature Space by Auto Encoder $(A E)$}

The basic structure of an AE is like a funnel, having an intermediate hidden layer. From input to the hidden layer, having reduced number of nodes, works as encoder i.e., reduces the feature space, followed by increasing number of nodes, working as the decoder. In this work the encoder part of the $A E$ is used to reduce the dimension of the feature space $[19,20]$ without any loss of information regarding classification. The objective of $A E$ is to manipulate the features in the hidden layer in such a way so that $X \approx \hat{X}$, such that, $X=\left[X^{j}\right] \in \Re^{(N \times p)}$, where $X, \hat{X}$ are the input at the encoder and output from the decoder of AE. Now corresponding to $X^{j}, q$ dimensional feature vector is extracted from the hidden layer as,

$$
Y^{j}=\phi\left(E_{1} \cdot X^{j}+b_{1}\right), \forall j
$$

where, $Y^{j} \in \Re^{(1 \times q)}$ is the compressed form of $X^{j}$,such that, $q<p$ and $E_{1}$ is the weight vector of unit connection between input layer and hidden layer of $\mathrm{AE}$, $b_{1}$ is the bias term associated to the hidden layer and $\phi($.$) is the nonlinear activation function of the AE.$

The hyper-parameters of $\mathrm{AE}\left(E_{1}, b_{1}\right)$ are updated to minimize the following objective function: 


$$
J\left(E_{1}, b_{1}\right)=\min \sum_{\forall j}\left[\hat{X}^{j}-X^{j}\right]^{2}
$$

Where, $\hat{X}^{j}$ is the decoder output of $\mathrm{AE}$ corresponding to input $X^{j}$.

\section{RESULTS}

In this present study on the chosen lower grades of brain tumours each parameters of the post-operative samples have been measured with their qualitative features and represented as absolute quantification values for the chosen biological parameters depicting the inflammatory-proliferative-invasive feature triangle.

\subsection{Histopathological Types and Grading}

The glioma types and grades were identified by histopathological analysis of samples in eosine- hematoxylin staining where four (4) samples identified with typical 'whorling' in meningioma, three (3) of the samples showed lobulated island with hyalinised fibrovascular core sometimes having perivascular pseudorosettes identifying as ependymoma and other three (3) samples showed higher number of glial cells having distinct fibrillary network indicating diffused fibrillary astrocytoma of lower grades.

\subsection{Inflammatory Status}

Inflammatory status of the glioma tissues with immune cell infestation and subsequent damage had been observed in three ways specially emphasising on the presence of brain macrophages and microglia in the neoplastic tissue. The silver-gold staining for brain macrophages and microglia were counted and
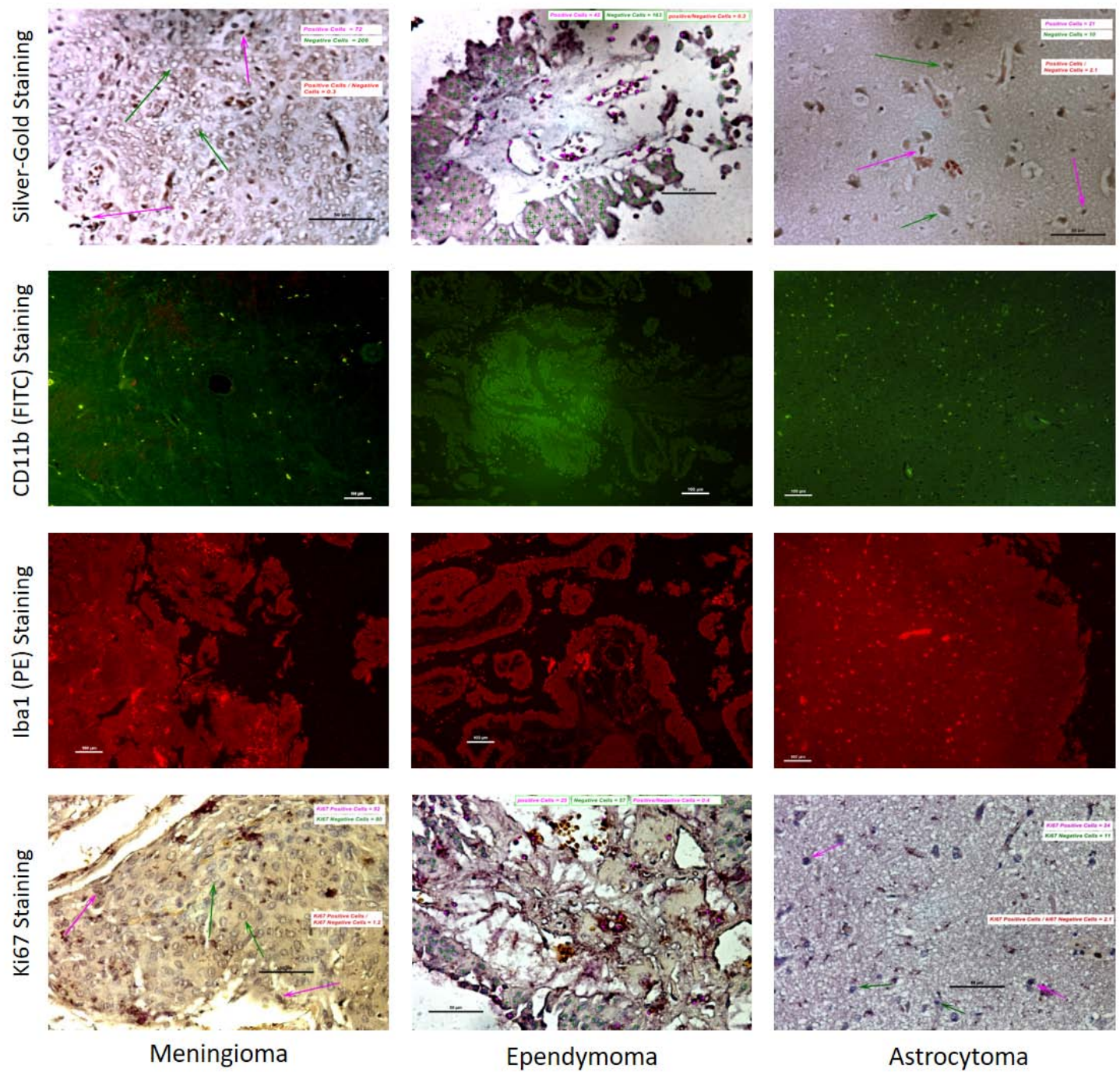

Figure 1: Representative microphotographs of biological attributes used for the machine learning applications. Identifiers of the panels are mentioned through the axis and quantification of such parameters are presented in Table 1. 
Table 1: Tabulated Values of Estimated Parameters for each Glioma Samples

\begin{tabular}{|c|c|c|c|c|c|c|c|}
\hline $\begin{array}{c}\text { Histopathological Types } \\
\text { of Glioma }\end{array}$ & Samples & $\begin{array}{c}\text { A: S-G } \\
\text { stained } \\
\text { cells }\end{array}$ & $\begin{array}{l}\text { B: CD11b } \\
\text { Intensity }\end{array}$ & $\begin{array}{l}\text { C: Iba1 } \\
\text { Intensity }\end{array}$ & $\begin{array}{l}\text { D: Ki67 } \\
\text { Index }\end{array}$ & $\begin{array}{c}\text { E: \% Proliferating } \\
\text { Cells }\end{array}$ & $\begin{array}{c}\text { F: MMP2 } \\
\text { MFI } \\
\text { Values }\end{array}$ \\
\hline \multirow[t]{4}{*}{ Meningioma } & $\mathrm{S} 1$ & 39 & 20.6 & 13.9 & 1.5 & 11.8 & 295 \\
\hline & $\mathrm{S} 2$ & 63 & 31.8 & 20.9 & 1.2 & 16.7 & 350 \\
\hline & S3 & 72 & 25.1 & 22.8 & 1.2 & 10.2 & 198 \\
\hline & S4 & 58 & 29.4 & 18.2 & 1.3 & 12.7 & 253 \\
\hline \multirow[t]{3}{*}{ Ependymoma } & $\mathrm{S} 1$ & 20 & 9.4 & 11.8 & 0.5 & 10.7 & 1358 \\
\hline & $\mathrm{S} 2$ & 32 & 15.4 & 10.6 & 0.4 & 6.1 & 1290 \\
\hline & S3 & 42 & 5.1 & 8.5 & 0.4 & 8.2 & 1443 \\
\hline \multirow[t]{3}{*}{ Astrocytoma } & $\mathrm{S} 1$ & 21 & 7.6 & 13.2 & 1.6 & 18.3 & 337 \\
\hline & $\mathrm{S} 2$ & 18 & 5.6 & 22.8 & 2.1 & 24.1 & 546 \\
\hline & S3 & 16 & 6.7 & 18.1 & 2.0 & 21.3 & 289 \\
\hline
\end{tabular}

compared among samples in equal areas tissues (Figure 1). Similarly the fluorescence intensity for CD11b expression for brain macrophages and Iba1 expression intensity for microglia in same areas of the samples quantified using NIS-BR software (Nikon Corp., Japan) and represented in Figure 1 and Table 1. Though a generalized weightage of presence of macrophages is found in meningioma, the value ranges for three methods in tumour tissue varies widely between individual samples, groups and methods with significant overlaps as tabulated.

\subsection{Proliferative Status}

To estimate the proliferation status of the samples, $\%$ of proliferating cells were measured by Propidium lodide (PI) staining followed by flowcytometric analysis by measuring the DNA content in $S$ and $G_{2} M$ phase of cell cycle and quantified data for each samples were tabulated in Table 1. The PI data of \% cell proliferation shows prominently three ranges of data distribution with minimal overlaps between tumour types. More specifically, the Ki67 positive cells were calculated as the indicator of proliferation and each tissue sample was indexed indicating the Ki67 positive and negative cell ratio (Figure 1) which showed similar distinctive ranges as of the previous parameter but with a continuity of data points among these three types. The results were given in Table 1.

\subsection{Invasive Status}

The invasive property of each type and stage of glioma was measured with the extracellular matrix (ECM) degrading gelatinase enzyme MMP2 expression. The mean fluorescence intensity of fluorescenated antibody against MMP2 protein expression for each samples were documented in flowcytometry and tabulated for quantification (Table 1) which shows mild overlap but wide distribution range of values.

\subsection{Graphical Representation of the Features}

The mentioned six parameters for all brain tumour samples are plotted individually, which has been shown in Figure 2. From the above plots it is clear that none of the feature can determine the stage of the Glioma individually, as many of the samples are having the overlapped values of parameters, though Ki67 data showing better separation than other parameters.

\subsection{Classification by Support Vector Machine Classifier}

Thus, to alleviate the problem of these overlapping values the multi class SVM is used. As the number of samples available was small, for the training and validation of the classifier LOO cross validation method was applied considering each of the samples as the test sample in some run out of its 10 runs. The performance of the proposed method is based on the following matrices.

True Positive $(T P)=\frac{\text { no. of samples of a given Glioma class correctly detected }}{\text { total number of samples in that given Glioma class }}$

False Positive $(F P)=\frac{\text { no. of samples of a given Glioma class detected as another class }}{}$ total number of samples in that given Glioma cla

Positive Predictivity $(P P)=\frac{T P}{(T P+F P)} \times 100 \%$ 


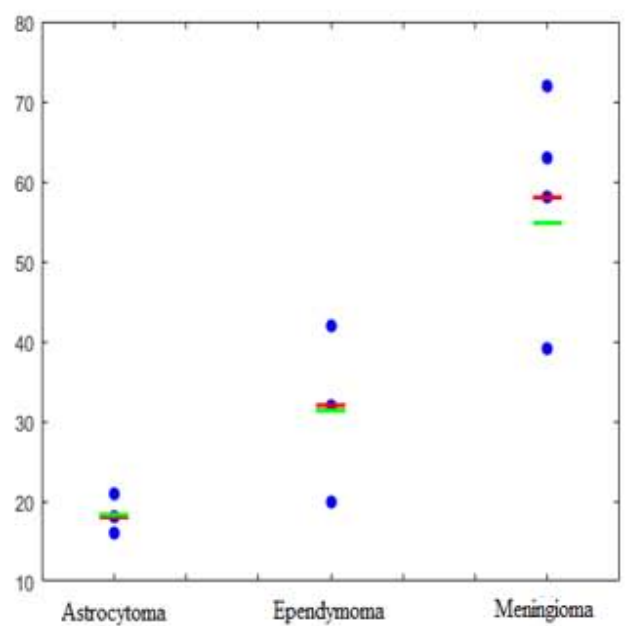

A: Silver-Gold Staining
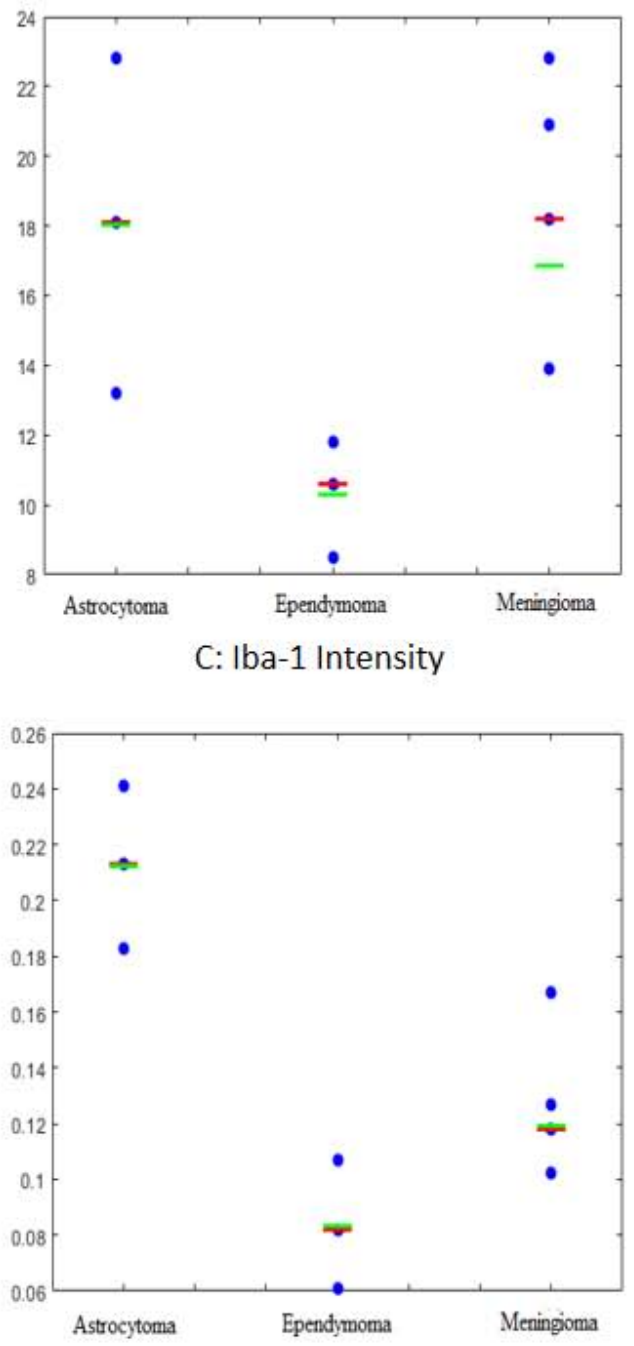

E: \% Cell Proliferation

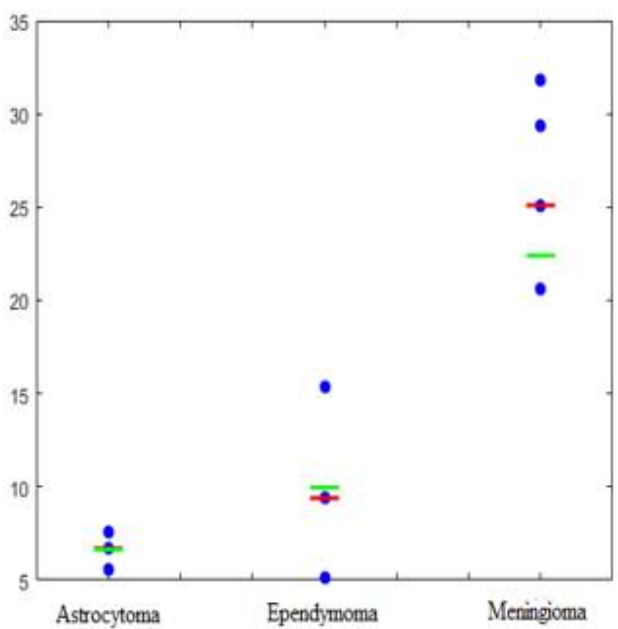

B: CD11b Intensity
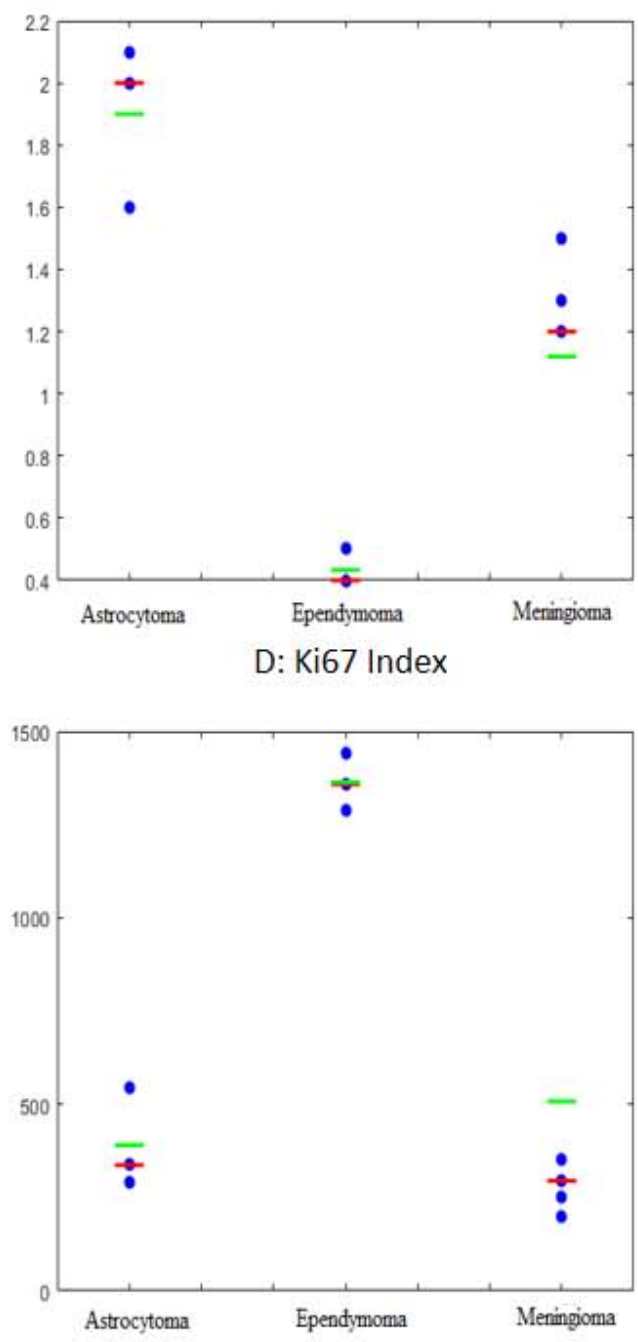

F: MMP2 MFI Value

Figure 2: A - F: Plot of each estimated parameter using support vector machine (SVM) classifier mentioned in the figure for all Brain Tumour samples. Green and Red bars represent mean and median values respectively. Plots are showing overlapping of features for the brain tumour types. In the plots, $1^{\text {st }}, 2^{\text {nd }}$ and $3^{\text {rd }}$ columns represent Astrocytoma, Ependymoma and Meningioma respectively. 
Table 2: Test Results of Classification of Samples using Positive Predictivity (PP) Test in Support Vector Machine (SVM)

\begin{tabular}{|c|c|c|c|c|}
\hline Types of Brain Tumour & No. of samples & TP & FP \\
\hline \hline Astrocytoma & 3 & 3 & 0 & $100 \%$ \\
\hline Ependymoma & 3 & 3 & 0 & $100 \%$ \\
\hline Maningioma & 4 & 4 & 0 & $100 \%$ \\
\hline
\end{tabular}

The test results have been shown in Table 2.

\subsection{Auto Encoder (AE) for 2D Reduced Feature Space}

Using $\mathrm{AE}$ the 6 dimensional feature space is reduced to 2 dimensional feature space without any loss of information regarding classification. This is shown in Figure 3. It is found that in the reduced dimensional feature/ latent space the nonlinearly separable problem has been converted to the linearly separable one. For AE there are 6 input nodes corresponding to 6 physically obtained features from each sample and the hidden layer consists of 2 nodes only which corresponds to the dimension of feature space.

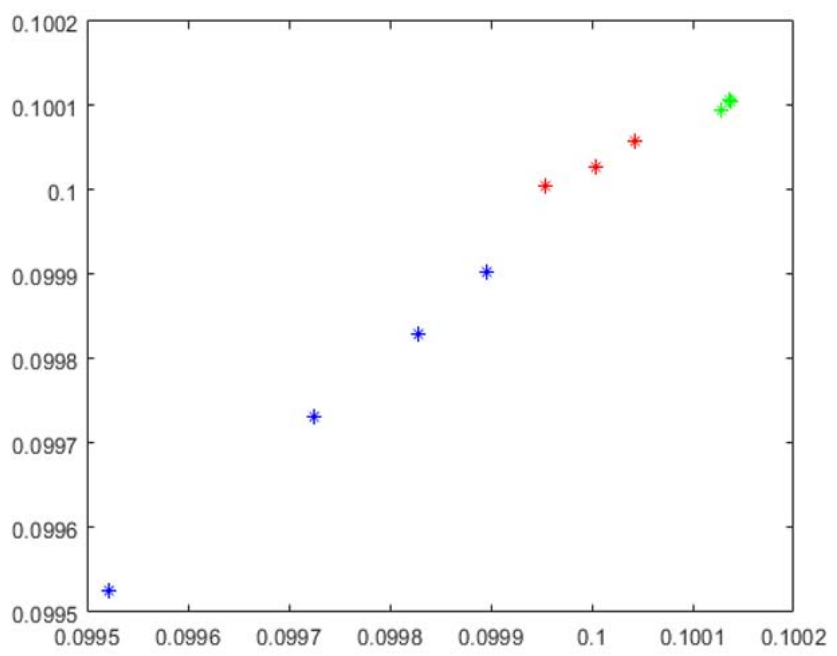

Figure 3: Plot of Glioma samples in the 2 dimensional reduced feature space using deep auto encoder (DAE). Blue dots represent the position of meningioma samples in that 2D featured space, where Red dots for Ependymoma and Green dots for Astrocytoma sample positions. Meningioma features being widely dispersed and Astrocytoma most clustered, however, none of the types of brain tumour features show overlap in contrast to SVM classification.

\section{DISCUSSION}

In the present study, depending on the histopathological classifications of the samples we measured the values of the mentioned six biological features of each glioma samples. The parameters chosen falls under three important axis of tumour hallmarks [1], namely, proliferation - invasion inflammation. While Ki67 indexing and cell cycle analysis with propidium iodide $(\mathrm{PI})$ indicated the proliferation status [15], MMP2 expression was meant for invasiveness owing to its direct connection with immune cell infestation in the glioma tissue [21, 22]. At the same time, the inflammatory hint about the samples were obtained from the presence of macrophage/microglia designated with silver-gold staining, CD11b+ and lba1+ cell presence as quantified by number or by fluorescence intensity of fluorescinated antibody [23,24]. In Figure 1, the immunohistochemical and immunofluorescent microphotographs of biological features of three selected low-grade tumour types are presented. Overall, these limited but key biological attributes of our limited sample size were evaluated through a machine learning process to test whether these samples can effectively be classified into separate tumour types depending on these hallmark features rather than based on merely histopathological structures found in tissue samples. If biological attributes can classify the sample types, it can be possible to identify probes to detect such features without depending upon the tissues of patients.

In India, some epidemiological studies indicated about the occurrence of glioma in the subcontinent including our local population. Overall, reporting found that over half of the brain tumour types are of glial origin, over $10-20 \%$ are meningioma and about $5 \%$ are ependymoma [25, 26]. But the problem is to detect brain tumour in early stages with their specific grades. So far, the actual diagnosis and grading depends on the histological studies after ablation of glioma mass, i.e, from post-operative tissue slides, which has no predictive diagnostic value. With practical experiences in hospital patients it is generally found that radiological predictive diagnosis success is low and sometime misleading. Complete and proper detection of Glioma type and grade is a challenge without histopathological slide preparation. We have tried to shift our diagnostic 
features to some key biological attributes related with neoplastic growth. Though the features are still dependent on ablated tissue, but such attempt showed possibilities to shift diagnostic measures on other biological features working in conjunction with machine learning applications, found capable to distinctly classify such brain tumour types. Not only that, we used very restricted sample size beyond the normal statistical acceptance level considering practical constrain of sample availability in some particular time and place with various obligations. Overcoming such constrains in sample size, the process fully satisfied the classification criteria and showed clustering of multiparametric data.

We primarily targeted low grade brain tumour types, as classifying and diagnosing tumours in lower grades are most challenging and effective from the perspective of prognostic measures. The multi class SVM were used to derive the results from the biological data sets. Studies show that none of the features individually fulfil the purpose and apparently produced varied data range with wide graphical representations (Table 1 and Figure 2). As it is difficult to visualize and realize a space more than 3 dimensions, the $A E$ has been used to reduce multidimensional space to 2 dimensions without any loss of information [19]. In the latent space, provided by the hidden layers of $A E$ the nonlinearly separable problem has become a linearly separable one. Since data were collected from only real brain tumour samples, it was impossible to measure the six given parameters for the healthy samples and thus, False Negative (FN), defined as percentage of healthy samples detected as any of the brain tumour classes and True Negative (TN), defined as percentage of healthy samples detected as healthy; could not be measured in this study. Thus it is also not possible to measure the sensitivity defined as the ratio of TP to $(T P+F N)$. To compensate, the leave one out (LOO) cross validation method has been applied which complement the sensitivity of the results in classifying the samples depending on the given biological features.

\section{CONCLUSION}

Present study showed that development of an efficient classifier for a type of cancer is possible by using some hallmark attributes of cancer rather than histopathological analysis. More precisely, ambiguities in pathological analysis for different post-operative or aspirated tumour samples which apparently possess overlapping ranges can be eliminated and these can be classified by combination of some other observed biological features using machine learning systems. In other words, clustering of combined characters from a sample giving rise to apparent overlaps of histopathological attributes inseparable by instinctive human cognitive capacity, can be removed and the inner distinctiveness is possible using the above mentioned machine learning applications by extracting the deeply encoded similarity patterns between sample groups. This gives rise to the opportunity to think about more accurate and unambiguous identification and their use for classifying not only brain tumours but also other tumours. This may probe to a newer diagnostic approach using machine interface in patho-clinical applications.

\section{ACKNOWLEDGEMENTS}

We are grateful to Prof. Samarendranath Ghosh of Department of Neurosurgery, Bangur Institute of Neurosciences (BIN), Institute of Post Graduate Medical Education and Research (IPGME\&R), Kolkata, India and M.Ch students at Neurosurgery unit, BIN, IPGME\&R for post-operative tumour samples. We also acknowledge Prof. Uttara Chatterjee of Department of Pathology, IPGME\&R, Kolkata for histopathological grading of the tumour samples, Dr. Ritesh Tiwari of BDCoE Flow Cytometry unit at CRNN, University of Calcutta for immuno-flowcytometry experiments and analysis, to Dr. Sanjaya Kumar Mallick, Director, Cytometry Solutions Pvt. Ltd, Kolkata for helping in cell cycle analysis from paraffin embedded tumour tissues.

\section{FUNDING DETAILS}

This work was supported by the Council of Scientific and Industrial Research (CSIR), Government of India for financial aid vide Sanction No. 37(1587)/13/EMR-II dated 01.04 .2013 to Anirban Ghosh as Principal Investigator $(\mathrm{PI})$ for the work.

\section{REFERENCES}

[1] Hanahan D, Weinberg RA. The hallmarks of cancer. Cell 2000; 100: 57-70.

https://doi.org/10.1016/S0092-8674(00)81683-9

[2] Hanahan D, Weinberg RA. Hallmarks of cancer: the next generation. Cell 2011; 144: 646-674. https://doi.org/10.1016/j.cell.2011.02.013

[3] Eckhouse S, Lewison G, Sullivan R. Trends in the global funding and activity of cancer research. Mol Oncol 2008; 2 : 20-32. https://doi.org/10.1016/j.molonc.2008.03.007

[4] American Brain Tumor Association (Official Home Page, USA). http: //www.abta.org/about-us/news/brain-tumor statistics [accessed on January 20, 2018] 
[5] Maldjian JA, Patel RS. Cerebral Neoplasms in adults. Semin Roentgenol 1999; 34(2): 102-22. https://doi.org/10.1016/S0037-198X(99)80025-X

[6] Bondy ML, Scheurer ME, Malmer B, et al. Brain Tumor Epidemiology: Consensus From the Brain Tumor Epidemiology Consortium. CANCER Suppl 2008; 113(7). 1953-68.

https://doi.org/10.1002/cncr.23741

[7] Duong LM, McCarthy BJ, McLendon RE, et al. Descriptive epidemiology of malignant and nonmalignant primary spinal cord, spinal meninges, and caudaequina tumors, United States, 2004-2007. CANCER 2012; 118: 4220-4227. https://doi.org/10.1002/cncr.27390

[8] Zitnik M, Nguyen F, Wang B, Leskovec J, Goldenberg A, Hoffman MM. Machine learning for integrating data in biology and medicine: Principals, practice and opportunities. Information Fusion 2019; 50: 71-91. https://doi.org/10.1016/j.inffus.2018.09.012

[9] Xu C, Jackson SA. Machine learning and complex biological data. Genome Biology 2019; $20: 76$. https://doi.org/10.1186/s13059-019-1689-0

[10] Shavers C, Li R, Lebby G. An SVM based approach to face detection. Proceedings of the $38^{\text {th }}$ Southeastern Symposium on System Theory, Tennessee Technological University Cookeville, TN, USA, IEEE 2006; pp. 362-366.

[11] Tutorial on Support Vector Machine (SVM) by Vikramaditya Jakkula, School of EECS, Washington State University.

[12] Comparative Study of Dimension Reduction Approaches With Respect to Visualization in 3-Dimensional Space, Pooja Chenna, Master's Thesis, Department of Computer Science Kennesaw State University, USA, May, 2016.

[13] Penfield W. A method of staining oligodendroglia and microglia (combined method). Am J Pathol 1928; 4: 153-157.

[14] Penfield W, Cone W. Neuroglia and microglia (the metallic methods). In: McClung CE, Paul B(ed) Handbook of Microscopical Techniques, Hoeber, New York, 1937; pp. 489-521.

[15] Jordanova ES, Corver WE, Vonk MJ, et al. Flow cytometric sorting of paraffin-embedded tumour tissues considerably improves molecular genetic analysis. Am J Clin Pathol 2003; 120: 327-334. https://doi.org/10.1309/HPR11R7LQ9NNCCG8

[16] Burgess CJC. A tutorial on support vector machines for pattern recognition. Data Min Knowl Disc 1998; 2: 955-974.
[17] Wu Y, Liu Y. Robust Truncated Hinge Loss Support Vector Machines. J Am Stat Assoc 2007; 102(479): 974-983. https://doi.org/10.1198/016214507000000617

[18] Elisseeff A, Pontil M. Leave-one-out error and stability of learning algorithms with applications Stability of Randomized Learning Algorithms Source, J Mach Learn Res; January, 2002.

[19] Zhu J, Wu L, Hao H, Song X, Lu Y. Auto-encoder based for high spectral dimensional data classification and visualization, in Proc. IEEE 2nd Int. Conf. Data Sci. Cyberspace, Shenzhen, China, Jun. 2017; pp. 350-354. https://doi.org/10.1109/DSC.2017.32

[20] Improving Photoplethysmographic Measurements Under Motion Artifacts Using Artificial neural Network for Personal Healthcare, M. Singha Roy, R Gupta, J. K. Chandra, K. Das Sharma, A. Talukdar, IEEE transaction on Instrumentation and Measurement 2018; 67(12). https://doi.org/10.1109/TIM.2018.2829488

[21] Snuderl M, Chi SN, De Santis SM, et al. Prognostic value of tumour microinvasion and metalloproteinases expression in intracranial pediatric ependymomas. J Neuropath Exp Neurol 2008; 67: 911-920. https://doi.org/10.1097/NEN.0b013e318184f413

[22] Rooprai HK, Rucklidge GJ, Panou C, et al. The effects of exogenous growth factors on matrix metalloproteinase secretion by human brain tumour cells. $\mathrm{Br} \mathrm{J}$ Cancer 2000; 82: $52-55$. https://doi.org/10.1054/bjoc. 1999.0876

[23] Ghosh K, Ghosh S, Chatterjee U, et al. Microglial contribution to glioma progression: An immunohistochemical study in Eastern India. Asian Pac J Cancer Prev 2016; 17: 27672773.

[24] Roggendorf W, Strupp S, Paulus W. Distribution and characterization of microglia/macrophages in human brain tumours. Acta Neuropathol 1996; 92: 288-293. https://doi.org/10.1007/s004010050520

[25] Ghosh A, Sarkar S, Begum Z, et al. The first cross sectional survey on intracranial malignancy in Kolkata, India: Reflection of the state of the art in southern West Bengal. Asian Pac J Cancer Prev 2004; 5: 259-267.

[26] Jaiswal J, Shastry AH, Ramesh A, et al. Spectrum of primary intracranial tumours at a tertiary care neurological institute: $A$ hospital-based brain tumour registry. Neurol India 2016; 64 : 494-501. https://doi.org/10.4103/0028-3886.181535

\section{https://doi.org/10.30683/1927-7229.2020.09.02}

(c) 2020 Ghosh et al.; Licensee Neoplasia Research.

This is an open access article licensed under the terms of the Creative Commons Attribution Non-Commercial License (http://creativecommons.org/licenses/by-nc/3.0/) which permits unrestricted, non-commercial use, distribution and reproduction in any medium, provided the work is properly cited. 